\title{
Aislamiento e identificación de bacterias tolerantes y bioacumuladoras de metales pesados, obtenidas de los jales mineros El Fraile, México Identification and isolation of heavy-metal tolerant and bioaccumulator bacteria obtained from El Fraile mine tailings, Mexico
}

\author{
Amairani Santana-Flores ${ }^{1}{ }^{(D)}$, Alejandro Sánchez-Ayala ${ }^{2}$ (D), Yanet Romero-Ramírez ${ }^{1}$ (i),

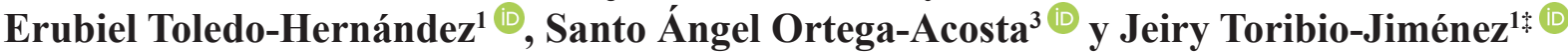

\footnotetext{
${ }^{1}$ Facultad de Ciencias Químico Biológicas, Universidad Autónoma de Guerrero. Av. Lázara Cárdenas s/n, Ciudad Universitaria Sur. 39087 Chilpancingo, Guerrero, México.

*Autor para correspondencia. (jeiryjimenez2014@gmail.com)

${ }^{2}$ Instituto Nacional de Investigaciones Forestales, Agrícolas y Pecuarias. CE-Zacatepec. Carretera Zacatepec Galeana s/n, Centro. 62780 Zacatepec de Hidalgo, Morelos, México.

${ }^{3}$ Facultad de Ciencias Agropecuarias y Ambientales, Universidad Autónoma de Guerrero. Carretera Iguala-Teloloapan s/n, Ignacio Manuel Altamirano. 40040 Iguala de la Independencia, Guerrero, México.
}

\section{RESUMEN}

La actividad minera ha dejado depósitos de jales ricos en metales pesados y metaloides. Por su toxicidad extrema, representan un ambiente hostil para la colonización microbiana; sin embargo, en estos residuos mineros existen bacterias metalotolerantes y esto se atribuye a mecanismos que destacan la bioacumulación y biotransformación de metales. Por lo tanto, las bacterias nativas que viven alrededor de los jales El Fraile, han desarrollado mecanismos de adaptación que las hace persistente en un ambiente tóxico; por tal motivo, el objetivo de este trabajo fue aislar e identificar bacterias capaces de tolerar, bioacumular o biotransformar metales pesados y metaloides. El estudio fue observacional descriptivo y se logró aislar 141 cepas de agua, lixiviados, suelo, rizosfera y tallos de plantas de los jales El Fraile, Guerrero, México. Se determinó tolerancia por concentración mínima inhibitoria en distintas sales metálicas, capacidad de bioacumulación de $\mathrm{Ag}, \mathrm{Pb}, \mathrm{Cr}, \mathrm{Cd}$ y biotransformación de As (III) y $\mathrm{Cr}^{6+}$; algunas bacterias se identificaron mediante la secuencia del gene 16S ARNr. Los resultados de las 141 cepas analizadas mostraron tolerancia ascendente a $\mathrm{Cd}^{2+} 2 \mathrm{mM}, \mathrm{Cu}^{2+} 5 \mathrm{mM}, \mathrm{Pb}^{2+}$ $9 \mathrm{mM}, \mathrm{Zn}^{2+} 9 \mathrm{mM}, \mathrm{Ag}^{2+} 10 \mathrm{mM}, \mathrm{As}^{3+} 30 \mathrm{mM}$ y As${ }^{5+}$ $45 \mathrm{mM}$. Solo 9.24 y $39 \%$ de las cepas bioacumularon

Cita recomendada:

Santana-Flores, A., A. Sánchez-Ayala, Y. Romero-Ramírez, E. ToledoHernández, S. Á. Ortega-Acosta y J. Toribio-Jiménez. 2020. Aislamiento e identificación de bacterias tolerantes y bioacumuladoras de metales pesados, obtenidas de los jales mineros El Fraile, México. Terra Latinoamericana 38: 67-75.

DOI: https://doi.org/10.28940/terra.v38i1.430
$\mathrm{Zn}^{2+}, \mathrm{Pb}^{2+}$ y $\mathrm{Ag}^{2+}$. En la biotransformación solo $9 \%$ oxidaron $\mathrm{As}^{3+} \mathrm{a} \mathrm{As}^{5+}, 7$ y $24 \%$ redujeron $\mathrm{As}^{5+} \mathrm{a} \mathrm{As}^{3+} \mathrm{y} \mathrm{Cr}^{6+}$ $\mathrm{a} \mathrm{Cr}^{3+}$, respectivamente. Los géneros que prevalecieron fueron Chryseobacterium sp., Staphyloccoccus sp., Stenotrophomonas sp., Acinetobacter sp., Bacillus sp., Serratia sp., y Enterobacter sp. Por lo tanto, existen cepas bacterianas en la zona minera El Fraile capaces de bioacumular y biotransformar metales pesados y metaloides; por lo que es necesario realizar más estudios que permitan conocer su potencial en los procesos de biorremediación de sitios contaminados con metales.

Palabras clave: Bacillus sp., biorremediación, contaminación, minería.

\section{SUMMARY}

The mining activity has left mine tailings with high quantities of heavy metals and metalloids. Due their extreme toxicity, they represent a hostile environment for microbial colonization; however, there are metalotolerant bacteria in these mining wastes, and this is attributed to mechanisms of bioaccumulation and biotransformation of metals. The native bacteria from El Fraile mine tailings have developed adaptation mechanisms that allow them to persist in a toxic environment, thus, the objective of this study was to 
isolate and identify bacteria with the ability to tolerate, bioaccumulate or biotransform heavy metals. Our study was based on descriptive observations, we isolated 141 strains from water, leachate, soil, and rhizosphere and stems of plants from El Fraile mine tailings, Guerrero, Mexico. Bacterial tolerance was determined by the minimum inhibitory concentration in different metallic salts, the capacity of bioaccumulation of $\mathrm{Ag}, \mathrm{Pb}, \mathrm{Cr}$, and $\mathrm{Cd}$, and by the biotransformation of As (III) and $\mathrm{Cr}^{6+}$; in addition, representative bacteria were identified using $16 \mathrm{~S}$ rRNA gene sequences. The results of the 141 strains analyzed showed ascending tolerance to $\mathrm{Cd}^{2+} 2 \mathrm{mM}, \mathrm{Cu}^{2+} 5 \mathrm{mM}, \mathrm{Pb}^{2+} 9 \mathrm{mM}, \mathrm{Zn}^{2+} 9$ $\mathrm{mM}, \mathrm{Ag}^{2+} 10 \mathrm{mM}, \mathrm{As}^{3+} 30 \mathrm{mM}$, and $\mathrm{As}^{5+} 45 \mathrm{mM}$. Only 9.24 and $39 \%$ of the strains bioaccumulated $\mathrm{Zn}^{2+}, \mathrm{Pb}^{2+}$ and $\mathrm{Ag}^{2+}$. In regard to biotransformation, 9\% oxidized $\mathrm{As}^{3+}$ to $\mathrm{As}^{5+}$, and 7 and $24 \%$ reduced $\mathrm{As}^{5+}$ to $\mathrm{As}^{3+}$ and $\mathrm{Cr}^{6+}$ to $\mathrm{Cr}^{3+}$, respectively. The genera that prevailed were Chryseobacterium sp., Staphyloccoccus sp., Stenotrophomonas sp., Acinetobacter sp., Bacillus sp., Serratia sp., and Enterobacter sp. These results showed that in El Fraile mine tailings, there are bacterial strains capable of bioaccumulating and biotransforming heavy metals and metalloids; therefore, further studies must be conducted to know their potential in the processes of bioremediation of areas polluted with metals.

Index words: Bacillus sp., bioremediation, pollution, mining.

\section{INTRODUCCIÓN}

La minería ha generado sitios altamente contaminados con metales pesados y metaloides, ocasionando que dicha actividad sea un problema ambiental con deterioro del suelo, contaminación de aire y agua, e impacto negativo en la diversidad $\mathrm{y}$ riqueza de plantas, insectos y vertebrados. La diversidad y abundancia microbiana en el suelo, y en la rizosfera de las plantas que sobreviven en esos sitios, también se deteriora (Tchounwou et al., 2012; RománPonce et al., 2016). La persistencia de los metales en el ambiente, causa efectos adversos a los ecosistemas, y la restauración ambiental, aún es muy costosa (Mosa et al., 2016). Una estrategia compatible con el ambiente es la biorremediación, la cual consiste en emplear organismos eucariotas o procariotas para restaurar áreas contaminadas. Diversos estudios con microorganismos metalotolerantes han demostrado remover, estabilizar o desintoxicar de una manera eficiente y menos costosa los sitios mineros abandonados (O'Brien y Buckling, 2015).

Los microorganismos, entre ellos las bacterias, han desarrollado la capacidad de sobrevivir en ambientes contaminados debido a su exposición constante a los compuestos tóxicos. Dentro de estas capacidades se incluyen la biosorción, bioacumulación y biotransformación de metales y metaloides, habilidades que pueden ser empleadas para la biorremediación ex situ o in situ de zonas perturbadas por las actividades de la minería. A la fecha, se han descrito géneros bacterianos capaces de tolerar, bioacumular y biotransformar metales pesados; de los que sobresalen Bacillus sp., Pseudomonas sp., Actinomyces sp., Serratia sp., Enterobacter sp., Thiobacillus sp., Rhodobacter sp., Agrobacterium sp., Acidiothiobacillus sp., entre otros (Costa y Duta, 2001; Hassan et al., 2008; El Baz et al., 2015).

Los jales El Fraile, Guerrero, México, están en la zona minera de Taxco, Guerrero, al sur de la Ciudad de México, tienen alrededor de 50 años a la intemperie, contienen residuos portadores de elementos potencialmente tóxicos (EPT) como $\mathrm{As}^{3+}, \mathrm{Pb}^{2+}, \mathrm{Cd}^{2+}$, $\mathrm{Tl}^{2+}, \mathrm{Hg}^{2+}, \mathrm{Cu}^{2+}, \mathrm{Fe}^{2+}, \mathrm{Mn}^{2+}, \mathrm{Co}^{2+}, \mathrm{Ni}^{2+}, \mathrm{Cr}^{6+}, \mathrm{V}^{2+} \mathrm{y} \mathrm{Zn}^{2+}$. Con volumen aproximado de 5.5 millones de toneladas de EPT, la peligrosidad de estos en el ambiente, está dada por la capacidad de generar lixiviados o drenaje ácido de mina (DAM), debido a la oxidación de la pirita que origina aguas ácidas con concentraciones altas de sulfatos, metales y metaloides en forma de iones disueltos que desembocan en el río Cacalotenango (Corrales-Pérez y Martín-Romero, 2013). TalaveraMendoza et al. (2006) cuantificaron en agua y lixiviados los contenidos de metales: $\mathrm{SO}_{4}^{2-}\left(280-29500 \mathrm{mg} \mathrm{L}^{-1}\right)$, As $\left(<0.01-12.0 \mathrm{mg} \mathrm{L}^{-1}\right)$, Fe $\left(0.025-2,352 \mathrm{mg} \mathrm{L}^{-1}\right)$, Mn (0.1-732 $\left.\mathrm{mg} \mathrm{L}^{-1}\right)$, Zn $\left(<0.025-1465 \mathrm{mg} \mathrm{L}^{-1}\right)$ y $\mathrm{Pb}$ $\left(<0.01-0.351 \mathrm{mg} \mathrm{L}^{-1}\right)$. El contenido de metales solubles en los jales son muy heterogéneos y se liberan: $\mathrm{Cd}^{2+}$, $\mathrm{Pb}^{2+}, \mathrm{Cu}^{2+}, \mathrm{Co}^{2+}, \mathrm{Zn}^{2+}$, y su acumulación en el suelo ha generado riesgo para los individuos que viven en las cercanías. Toribio-Jiménez et al. (2014) aislaron cepas de El Fraile tolerantes a $\mathrm{Cd}^{2+}, \mathrm{Pb}^{2+}, \mathrm{Cu}^{2+}, \mathrm{Zn}^{2+}, \mathrm{y} \mathrm{As}^{3+}$, las cuales las identificaron como Enterobacter sp., Pseudomonas sp., Staphylococcus sp., y Bacillus sp. Las bacterias con capacidad de bioacumular y biotransformar metales pesados y metaloides, son la base para el establecimiento de nuevas alternativas de biorremediación. La hipótesis del presente estudio 
señala que las bacterias presentes en los alrededores de los jales El Fraile han desarrollado mecanismos de adaptación para sobrevivir en presencia de metales pesados y metaloides; capacidades que se pueden aprovechar para diseñar estrategias de biorremediación de zonas mineras. El objetivo del trabajo se centró en aislar e identificar bacterias capaces de bioacumular $\mathrm{Ag}, \mathrm{Cd}, \mathrm{Cr}, \mathrm{Pb}$ y $\mathrm{Zn}$ y biotransformar $\mathrm{Cr}^{6+} \mathrm{a} \mathrm{Cr}^{3+}, \mathrm{As}^{5+}$ $\mathrm{a} \mathrm{As}^{3+} \mathrm{o} \mathrm{As}^{3+} \mathrm{a} \mathrm{As}^{5+}$, estás fueron obtenidas a partir de muestras de suelo, agua, lixiviados, rizosfera, y tallos de algunas especies de plantas silvestres.

\section{MATERIALES Y MÉTODOS}

Aislamiento de bacterias nativas. Se realizó un estudio observacional descriptivo a partir de 125 muestras (25 por cada matriz ambiental) tomadas de diferentes ambientes; suelo, agua, lixiviados, rizosfera, y tallos de plantas silvestres de los alrededores de los jales El Fraile, Taxco de Alarcón, en Guerrero. Las muestras de suelo se tomaron a una distancia aproximadamente de $50 \mathrm{~m}$ de los jales, el agua fue tomada del caudal del río Cacalotenango donde descargan los lixiviados de los jales, los lixiviados fueron tomados de las pozas que había, formadas sobre los jales; en cuanto a la raíz y tejido de las plantas se tomaron 4 especímenes de cada una en las que destacan; Acacia farnesiana, Hypericum perforatum, Paspalum sp., Pityrogramma sp., Prosopis laevigata, Lysiloma sp., según especies reportadas por Gómez-Bernal et al. (2010). Todas las muestras se transportaron, en bolsas plásticas o frascos estériles al laboratorio. Para el aislamiento de bacterias se tomó $1 \mathrm{~g} \mathrm{o} 1 \mathrm{~mL}$, de cada muestra, y se prepararon diluciones de $10^{-1}$ a $10^{-6}$, se inocularon $100 \mu \mathrm{L}$ de la última dilución en agar LB por triplicado, se incubaron a $30^{\circ} \mathrm{C}$ por $24-48 \mathrm{~h}$, las colonias fueron seleccionadas con base a sus características macro y microscópicas (Yu et al., 2014). Todos los experimentos se hicieron por triplicado y se expresaron en $\mathrm{UFC} \mathrm{mL}^{-1} \mathrm{o} \mathrm{g}$ de muestra.

Determinación de la CMI a metales pesados y metaloides. En cajas de agar LB se adicionaron sales metálicas; $\mathrm{Pb}\left(\mathrm{NO}_{3}\right)_{2}, \mathrm{Zn}\left(\mathrm{NO}_{3}\right)_{2}, \mathrm{Cd}\left(\mathrm{NO}_{3}\right)_{2}, \mathrm{Cu}\left(\mathrm{NO}_{3}\right)_{2}$, $\mathrm{NaAsO}_{2}$, y $\mathrm{NaH}_{2} \mathrm{AsO}_{4}, \mathrm{AgNO}_{3}$, en concentraciones de ascendentes que van de 0.5 a $20 \mathrm{mM}$. La CMI se interpretó como la concentración más alta de sales en la cual no se observó crecimiento después de 7 días de exposición (Yu et al., 2014; Román-Ponce et al., 2016).
Detección de la capacidad de bioacumular metales. Todas las cepas se inocularon en agar LB a la concentración entre 0.5 a $20 \mathrm{mM}$ dependiendo de la sal metálica siguiendo el protocolo e interpretación. Posteriormente, solo se seleccionó una cepa para confirmar la bioacumulación de $\mathrm{Pb}, \mathrm{Cr}$ y $\mathrm{Cd}$, por microscopia electrónica de transmisión (JOEL JEM1010 TEM) en el Instituto de Biotecnología de la UNAM (Guerrero-Jiménez et al., 2013; CastellanosMendoza et al., 2014).

Biotransformación de arsénico y cromo. Para la detección de bacterias biotransformadoras de arsénico; se inocularon en caldo LB durante $120 \mathrm{~h}$ a $29^{\circ} \mathrm{C}$, se centrifugaron a $3400 \mathrm{rpm}$ durante $30 \mathrm{~min}$, se lavaron con SSI estéril y se resuspendieron en $2 \mathrm{~mL}$ de la misma solución. Se inoculó $1 \mathrm{~mL}$ de la suspensión en $5 \mathrm{~mL}$ de caldo LB adicionado con $\mathrm{As}^{3+} \mathrm{y} \mathrm{As}^{5+}$ incubado a $29{ }^{\circ} \mathrm{C}$ por $120 \mathrm{~h}$. En una microplaca se añadieron $150 \mu \mathrm{L}$ del inóculo y $150 \mu \mathrm{L}$ de $\operatorname{AgNO}_{3}(0.1 \mathrm{M})$ a cada pocillo. Un resultado positivo se interpretó con base a la reacción del inóculo con $\mathrm{AgNO}_{3}$ formándose una coloración amarilla en $\mathrm{As}^{5+}$ y café en $\mathrm{As}^{3+}$. Como control negativo se utilizó caldo $\mathrm{LB}$ con $\mathrm{NaAsO}_{2}$ y $\mathrm{NaH}_{2} \mathrm{AsO}_{4}$ sin inocular (Simeonova et al., 2004). En el caso de la biotransformación del cromo; las cepas bacterianas fueron inoculadas en caldo LB suplementado con $\mathrm{Cr}^{6+}$ durante $120 \mathrm{~h}$ a $29^{\circ} \mathrm{C}$. Se agregó $1.5 \mathrm{~mL}$ de difenilcarbanizada, el $\mathrm{Cr}^{6+}$ contenido en cada sobrenadante se determinó mediante la medición de la absorbancia a $540 \mathrm{~nm}$. El resultado positivo se interpretó con base a la concentración de $\mathrm{Cr}^{6+}$ en el tubo inoculado con cada una de las cepas y control negativo se utilizó caldo $\mathrm{LB}$ adicionado con $\mathrm{Cr}^{6+}$ sin inocular (Verma et al., 2009).

Identificación molecular y filogenia de las bacterias metalotolerantes. El DNA de las cepas se extrajo de acuerdo al protocolo de Arnold y Lutzoni (2007). Después, la amplificación se realizó por PCR del gene $16 \mathrm{~S}$ ARNr, en un volumen final de $25 \mu \mathrm{L}$ siguiendo el protocolo de Weisburg et al. (1991). Los productos de PCR fueron limpiados, cuantificados, normalizados y secuenciados directamente (Applied Biosystems BigDye Terminator v3.1 cycle sequencing kit) en la Universidad de Arizona (Genetics Core. Bidirectional sequencing was performed on an Applied Biosystems 3730xl DNA Analyzer) (Foster City, CA, USA) (Hoffman y Arnold, 2010). El consenso de secuencias se realizó por ChromaSeq (Maddison y Maddison, 
2005), implementado en Mesquite v.2.6 (Maddison y Maddison, 2009), los análisis filogenéticos se realizaron en MEGA v. 7.0, se agruparon en unidades taxonómicas operacionales (OTU) y se depositaron al GenBank (https://www.ncbi.nlm.nih.gov/genbank/).

Estadística. Los datos fueron analizados mediante ANOVA de una vía, comparando las medias mediante el método Holm-Sidak, Sigma Plot 11.0. El nivel de significancia fue con valor de $P=0.05$. Los experimentos se realizaron con tres réplicas y tres repeticiones para cada uno.

\section{RESULTADOS Y DISCUSIÓN}

Aislamientos de cepas nativas. De los jales mineros El Fraile se aislaron 141 cepas basadas en las características macro y microscópica, aisladas de suelo, rizosfera, tallos, agua y lixiviados respectivamente (Figura 1, Cuadro 1). La diversidad bacteriana en los sitios está controlada por diversos factores bióticos y abióticos de cada hábitat que favorecen su establecimiento y crecimiento; por lo cual es favorecida en suelo y rizosfera por la disposición de nutrientes. Los géneros de Bacillus y Pseudomonas en suelo y rizosfera, se adaptan más fácilmente a los cambios de temperatura, $\mathrm{pH}$ y desecación entre otros, y la selección de resistencia a sustancias tóxicas que les permiten sobrevivir en sitios contaminados con metales, los cuales representan un sitio hostil para su crecimiento (Yu et al., 2014). Así mismo, las asociaciones entre planta-microorganismo-suelo se dan por medio de interacciones químicas, donde las plantas atraen a diferentes poblaciones microbianas a su rizosfera para su colonización (Schlatter et al., 2015). Mientras que en los tallos, agua y lixiviados representan un ambiente poco propicio para la colonización de bacterias, debido a la cantidad de nutrientes y al $\mathrm{pH}$ ácido en lixiviados. Lo anterior concuerda con los resultados obtenidos en este estudio, donde el mayor número de cepas aisladas corresponden a suelo y rizosfera. Román-Ponce et al. (2016) evaluaron la interacción entre bacterias endófitas de plantas y la contaminación por As en Prosopis laevigata y Sphaeralcea angustifolia en sitios contaminados en San Luis Potosí, México, destacando seis aislamientos con tolerancia a $\mathrm{As}^{5+}$ y $\mathrm{As}^{3+}$, y concuerdan que la diversidad de las comunidades endófitas es afectada por las concentraciones y el tipo de metal asociado. a

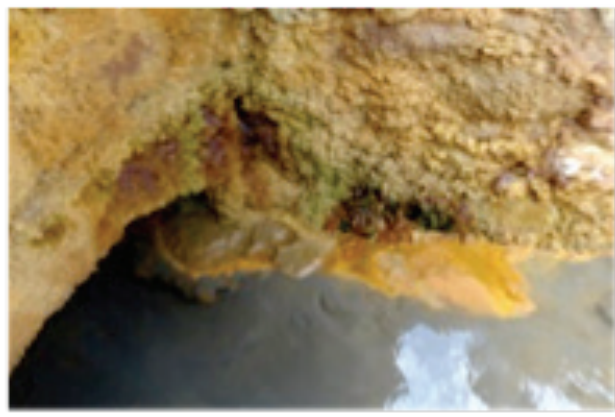

c

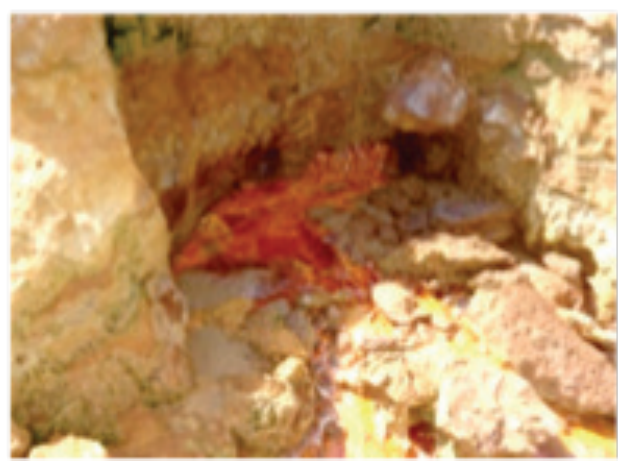

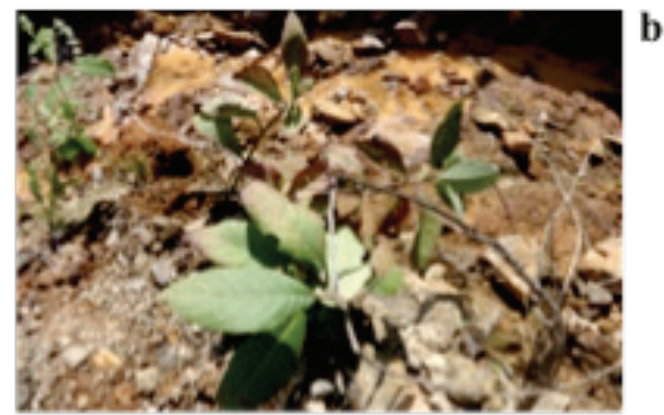

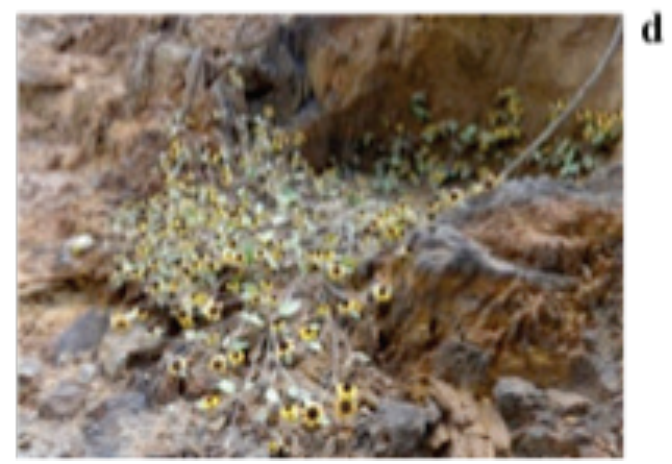

Figura 1. Sitios de muestreo en los jales El Fraile, a) agua, b) suelo, c) lixiviados y d) rizosfera. (fuente: LMM y BA).

Figure 1. Sampling sites in mine tailings from EI Fraile, a) water, b) soil, c) leachate, and d) rhizosphere. 
Cuadro 1. Características generales de la comunidad bacteriana tolerantes a metales pesados aislada de diversa matriz ambiental de los jales El Fraile, Taxco de Alarcón, Guerrero.

Table 1. Characteristics of the heavy-metal tolerant bacterial community isolated from the environmental matrix of El Fraile mine tailings, Taxco de Alarcón, Guerrero.

\begin{tabular}{|c|c|c|c|c|}
\hline Muestra & $\begin{array}{c}\text { Bacterias aisladas } \\
\text { por muestra }\end{array}$ & $\begin{array}{l}\text { CMI de los metales } \\
\text { evaluados }\end{array}$ & $\begin{array}{l}\text { Cepas tolerantes a cada } \\
\text { uno de los metales }\end{array}$ & Géneros identificados \\
\hline & $\%$ & $\mathrm{mM}$ & $\%$ & \\
\hline Suelo & 41 & $\mathrm{~Pb}[8 \mathrm{mM}]$ & 52 & Chryseobacterium sp. \\
\hline Rizosfera & 33 & $\mathrm{Cu}[4 \mathrm{mM}]$ & 80 & Staphylococcus sp. \\
\hline Tallo & 16 & $\mathrm{Cd}[1 \mathrm{mM}]$ & 57 & Pseudomonas sp. \\
\hline Agua & 7 & $\operatorname{Ag}[10 \mathrm{mM}]$ & 71 & Stenotrophomonas sp. \\
\hline \multirow[t]{3}{*}{ Lixiviados } & 3 & $\mathrm{Cr}[1 \mathrm{mM}]$ & 45 & Enterobacter sp. \\
\hline & & As (III) $[30 \mathrm{mM}]$ & 45 & Bacillus sp. \\
\hline & & As (IV) $[45 \mathrm{mM}]$ & 94 & Serratia sp. \\
\hline
\end{tabular}

Concentración mínima inhibitoria a metales pesados y metaloides. En la determinación de la CMI se observaron cambios en la morfología y pigmentación de las colonias en función a la exposición del metal (Figura 2), particularmente las cepas aisladas de agua, suelo y rizosfera presentaron cambios en presencia de $\mathrm{Pb}^{2+}$ y $\mathrm{Zn}^{2+}$; sin embargo, el mismo fenotipo se observó en $S$. aureus cuando fue expuesta a $\mathrm{HgCl}_{2}$. La CMI para cada uno de los metales evaluados en las 141 cepas se presentan en el Cuadro 1. Los resultados obtenidos en este estudio son similares a los de Moraga et al. (2003), con respecto a Pseudomonas sp., y Alcaligenes sp., donde $100 \%$ de las cepas fueron tolerantes a $\mathrm{Pb}^{2+} \mathrm{y}$ $\mathrm{As}^{3+}, 77.7 \%$ a $\mathrm{Cu}^{2+}$ y $36.4 \%$ a $\mathrm{Ni}$.

Los géneros de Acetobacter, Acinetobacter, Citrobacter, Enterobacter, Erwinia, Escherichia, Methylococcus, Xanthomonas, Vibrio, Bacillus, Micrococcus,
Planococcus, Staphylococcus, Streptomyces y Pseudomonas; también se describen como tolerantes a $\mathrm{Cu}^{2+}, \mathrm{Cd}^{2+}, \mathrm{Hg}^{2+}, \mathrm{Mn}^{2+}, \mathrm{Ni}^{2+}$ y $\mathrm{Zn}^{2+}$ (Altalhi, 2009; Martínez et al., 2010; Heck et al., 2015). Las bacterias aisladas de la rizosfera en las plantas silvestres de los jales El Fraile toleran mayores concentraciones de metales que las aisladas de los lixiviados y se debe a que los metales se encuentran disponibles por el $\mathrm{pH}$ ácido que presentan; algunos de estos géneros concuerdan con los reportados por Toribio-Jiménez et al. (2014) en los jales El Fraile tales como; Enterobacter, Klebsiella, Solibacterium. Los reportes anteriores sugieren que las bacterias pueden subsistir en ambientes diversos, perturbados por metales pesados y metaloides en diversas concentraciones y por ende han aprendido a tolerar su efecto con la finalidad de persistir como especie.
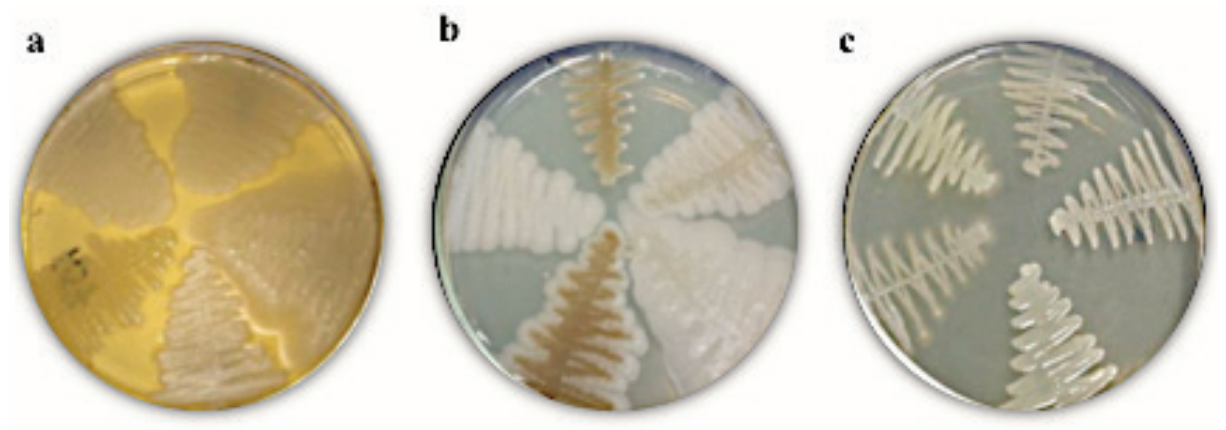

Figura 2. Ejemplos de los cambios de la morfología colonial de las cepas en presencia y ausencia de metales. a) Control (-) sin metal, b) $\mathrm{Pb}^{2+}$ y c) $\mathrm{Zn}^{2+}$.

Figure 2. Examples of morphological changes of bacterial strains in the presence and absence of heavy metals. a) (-) control without metals, b) $\mathrm{Pb}^{2+}$ y c) $\mathrm{Zn}^{2+}$. 
Capacidad de bioacumulación de metales pesados. La tolerancia a metales pesados está mediada por diferentes mecanismos celulares; entre ellos, la capacidad de expulsar, bioacumular y biotransformar los metales pesados y metaloides entre otros (MarreroCoto et al., 2010). Los genes de resistencia a As están codificados en dos operones ( $\operatorname{ars} R D A B C$ y $\operatorname{ars} R B C)$. El operón ars $R D A B C$ se ha descrito en plásmidos en E. coli R773 (Saltikov y Olson, 2002), el operón arsRBC en cromosoma o plásmidos de $S$. aureus pI258 (Ji y Silver, 1992). El arsR codifica para una proteína metaloreguladora (ArsR), y modula la sobreexpresión de $\operatorname{ars} R$ durante la bioacumulación del arsénico, $\operatorname{ars} B$ codifica para proteínas en la membrana plasmática que conforman bombas de expulsión, las cuales exportan al arsenito del citoplasma hacia el exterior de la célula, mientras ars $C$ codifica proteínas que reducen el arsenato a arsenito (Yang et al., 2015; Andres y Bertin, 2016).

Las bacterias presentes en los alrededores de los jales mineros El Fraile, se observa bioacumulación en 39\% de $\mathrm{Ag}^{2+}, 24 \% \mathrm{~Pb}^{2+}$ y $9 \%$ de $\mathrm{Zn}^{2+}$ respectivamente (Figura 3), esta capacidad se correlaciona a que estos metales están en mayor abundancia en el sitio en concentraciones para $\mathrm{Ag}^{2+}$ de $9.5-74.2 \mathrm{mg} \mathrm{kg}{ }^{-1}, \mathrm{~Pb}^{2+}$ de 780.43-700 mg kg-1 y $\mathrm{Zn}^{2+}$ de 380-10 $000 \mathrm{mg} \mathrm{kg}^{-1}$ según lo reportado por Talavera-Mendoza et al. (2005). A diferencia de este estudio, Costa y Duta (2001) describen cepas de B. sphaericus, B. cereus y B. subtilis, bioacumuladoras de $\mathrm{Cu}^{2+}, \mathrm{Zn}^{2+}$, y $\mathrm{Cd}^{2+}$. En el estudio de El Baz et al. (2015) aislaron 59 cepas de actinobacterias en áreas de minas abandonadas, todas fueron capaces de bioacumular $\mathrm{Pb}^{2+}$. $\mathrm{La}$ sobrevivencia de las cepas en ambientes altamente tóxicos es posiblemente en gran medida a su capacidad de bioacumular y biotransformarlos. En este estudio logró identificar cepas de los géneros de Enterobacter (rizosfera), Stenotrophomonas y Acinetobacter (agua), capaces de bioacumular $\mathrm{Ag}^{2+}$ y Zn ${ }^{2+}$, y Staphylococcus solo bioacumula $\mathrm{Ag}^{2+}$ (rizosfera). De los géneros identificados solo se encontraron reportados para Enterobacter de biocumular $\mathrm{Zn}^{2+}$ (Marrero-Coto et al., 2010), en cuanto a Stenotrophomonas, Staphylococcus y Acinetobacter, no se encontraron reportes con esa capacidad. Los jales El Fraile por sus características es un ambiente que induce, selecciona y dota a las bacterias nativas con mecanismos para tolerar la toxicidad de metales.

Biotransformación de arsénico. De las 141 cepas, 9\% muestran la capacidad de oxidar $\mathrm{As}^{3+} \mathrm{aAs}^{5+}$ y $6 \%$ reducir $\mathrm{As}^{5+} \mathrm{a} \mathrm{As}^{3+}$ esto fue observado en mayor proporción en las bacterias aisladas de rizosfera, seguido por las de suelo y lixiviados. Drewniak y Sklodowska (2013) reportaron en suelos mineros a $P$. azotoformans y $A$. ferroxidans capaz de oxidary reducir As y Pandey y Bhatt (2015) reportan a Exiguobacterium sp., As-9 capaz de biotransformar y remover As hasta en 99\% en China, y se atribuye a la posible actividad de enzimas arseniato reductasa, y arsenito oxidasa y arseniato reductasa citoplasmática que facilitan la biotransformación del As, como un mecanismo de detoxificación. Nunes et al. (2014), reportan la eficiencia de arseniato reductasa en Desulfovibrio alaskensis G20, todo esto es válido dado que diversas publicaciones comentan que la exposición
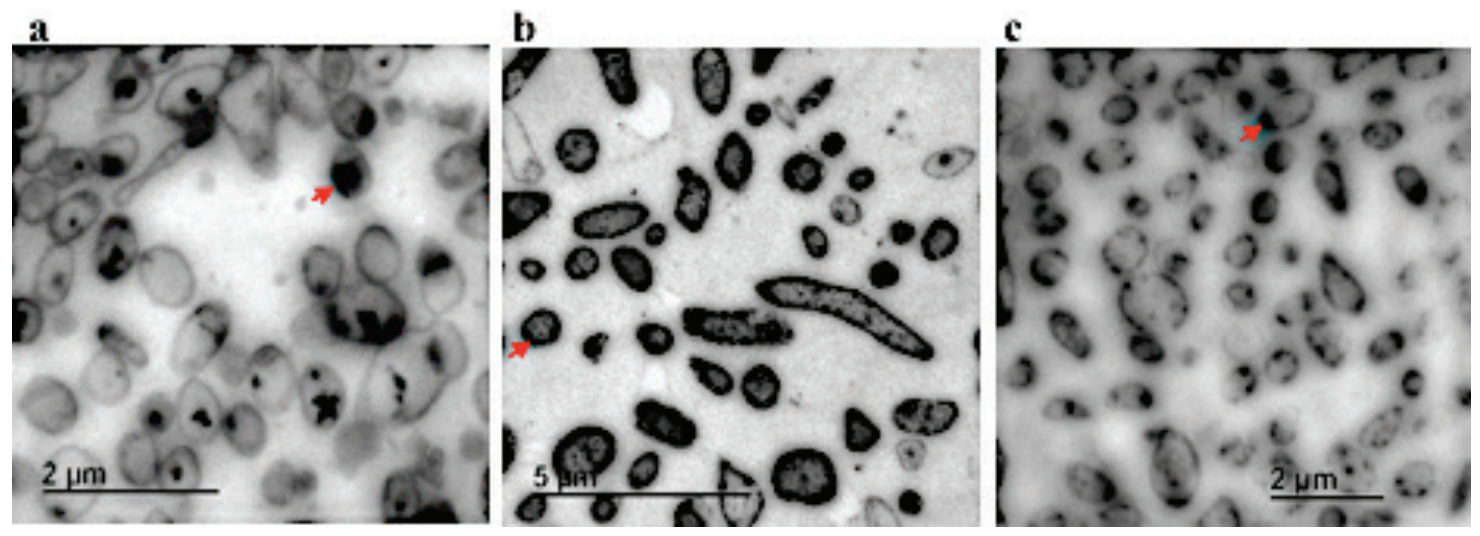

Figura 3. Bioacumulación de metales pesados por TEM en una cepa JM01 aislada de rizosfera. a) $\mathrm{Pb}$, b) $\mathrm{Cr}$, y c) Cd.

Figure 3. Heavy metal bioaccumulation by TEM of JM01 strain isolated from the rhizosphere. a) $\mathrm{Pb}$, b) $\mathrm{Cr}$, and c) Cd. 
al As de las bacterias nativas en sitios contaminados tienen la capacidad de oxidarlo o reducirlo.

Biotransformación de cromo. Solo $24 \%$ de todas las bacterias en El Fraile reducen el $\mathrm{Cr}^{6+}$, y las de rizosfera lo biotransforman en 64\%. Poornima et al. (2010) reportó una cepa de Pseudomonas sp., con capacidad de reducir el $\mathrm{Cr}^{6+}$ hasta $97.8 \%$ partiendo de una concentración inicial de $100 \mu \mathrm{g} \mathrm{mL} \mathrm{m}^{-1}$, en $24 \mathrm{~h}$ a $\mathrm{pH}$ de 5.0. Oves et al. (2013), reportan la reducción de 1800 $\mu \mathrm{g} \mathrm{mL} \mathrm{m}^{-1}$ del metal en $P$. aeruginosa OSG41 en $120 \mathrm{~h}$ en $\mathrm{pH}$ de 6-8., y B. brevis redujo $78.5 \%$ (Verma et al., 2009), la reducción microbiana del $\mathrm{Cr}^{6+}$ es catalizada por enzimas solubles, excepto en P. maltophila O-2 y $B$. megaterium TKW3, que utilizan reductasas asociadas a la membrana (Cheung y Gu, 2007). La reducción significativa del $\mathrm{Cr}^{6+}$ a $\mathrm{Cr}^{3+}$ es importante porque transforma al Cr tóxico y móvil en especies reducidas inocuas e inmóviles, fundamental para los procesos de biorremediación (Ahemad, 2014).

Identificación molecular y filogenia de bacterias metalotolerantes. Los géneros bacterianos identificados corresponden a Chryseobacterium, Staphylococcus, Acinetobacter, Stenotrophomonas, Pseudomonas, Enterobacter, Bacillus, y Serratia (Cuadro 1 y Figura 4). Por otra parte, Yu et al. (2014) aislaron 136 cepas de suelo de la mina V-Ti magnetita en China e identificaron que el mayor número de aislamientos fueron B. subtilis (32), B. pumilus (14), Rhizobium sp. (29) у O. intermedium (13).

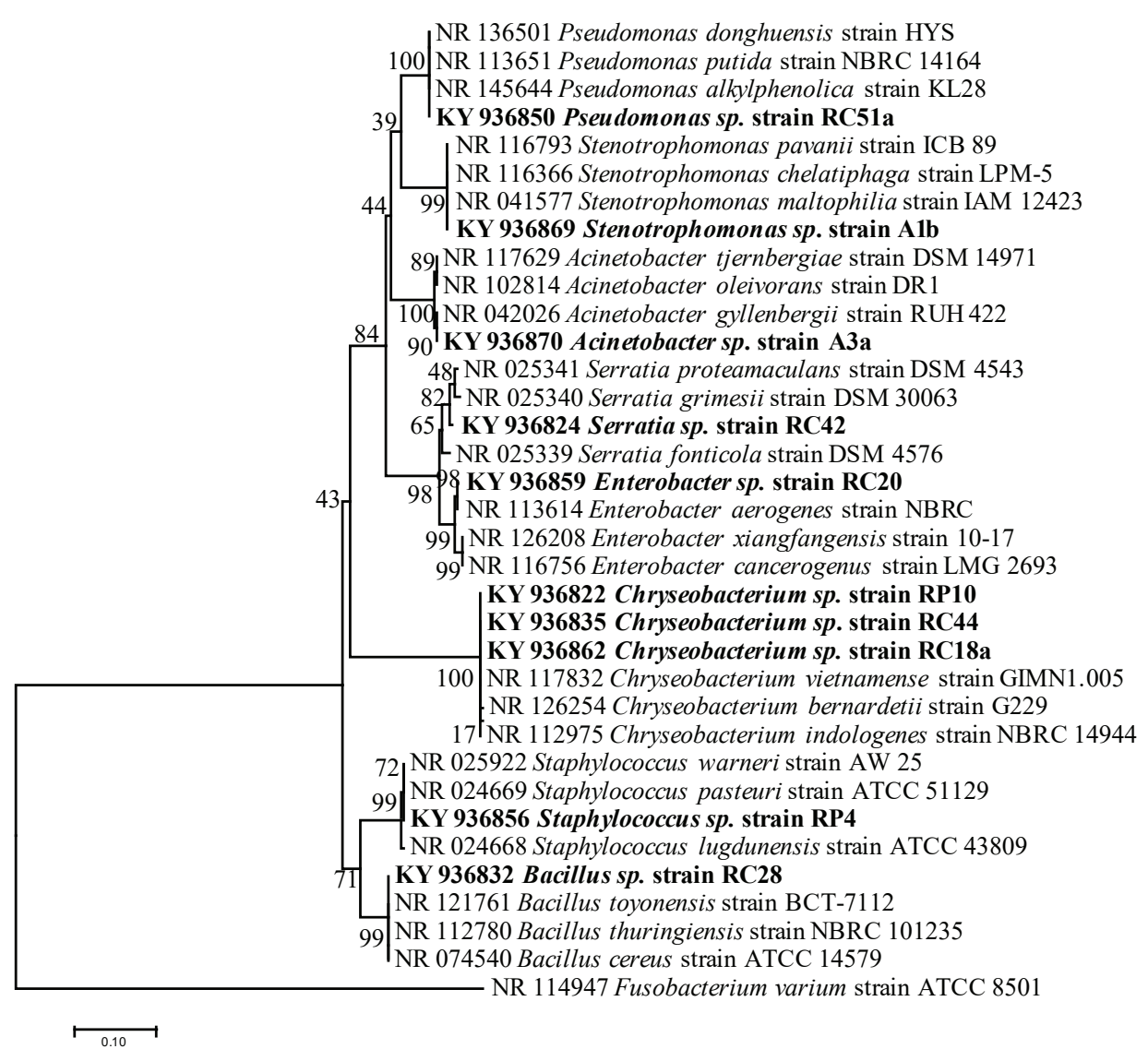

Figura 4. Árbol filogenético obtenido a partir de la secuencia de nucleótidos del gen $16 \mathrm{~S}$ ARNr de 10 aislados bacterianas de los jales mineros El Fraile (negritas). Fusobacterium varium (ATCC 8501) fue incluida como grupo externo. El árbol se construyó utilizando el método Neighbor-Joining con 1000 réplicas de Bootstrap (Saitou y Nei, 1987).

Figure 4. Phylogenetic tree obtained from 16S rRNA gene sequences of ten bacterial isolates from El Fraile mine tailings (bold type). Fusobacterium varium (ATCC 8501) was included as outgroup. The tree was built using the neighbor-joining method with 1000 bootstrap replications (Saitou and Nei, 1987). 


\section{CONCLUSIÓN}

Se aislaron 141 cepas bacterianas de agua, lixiviados, suelo, rizosfera y tallos de plantas de los jales El Fraile, Guerrero, México. Estás bacterias demostraron capacidad de tolerar, acumular y biotransformar diferentes metales pesados y metaloides. La identificación molecular demostró que pertenecen a los géneros Chryseobacterium sp., Staphyloccoccus sp., Stenotrophomonas sp., Acinetobacter sp., Bacillus sp., Serratia sp., y Enterobacter sp. Estas bacterias han demostrado poseer características importantes que se requieren para la biorremediación de zonas altamente contaminadas por actividades mineras, es necesario realizar más trabajos que permitan conocer su potencial en los procesos de biorremediación de sitios contaminados.

\section{AGRADECIMIENTOS}

Agradecemos a la Universidad de Arizona por la secuenciación de las cepas de este estudio, Abraham Morales y José Luis Aguirre Noyola por su asistencia técnica y la Unidad de Microscopía Electrónica del IBT, UNAM por las fotografías de la microscopia.

\section{LITERATURA CITADA}

Ahemad, M. 2014. Bacterial mechanisms for Cr (VI) resistance and reduction: an overview and recent advances. Folia Microbiol. 59: 321-332. doi: https://doi.org/10.1007/s12223-014-0304-8.

Altalhi, A. D. 2009. Plasmids profiles, antibiotic and heavy metal resistance incidence of endophytic bacteria isolated from grapevine (Vitis vinifera L.). Afr. J. Biotechnol. 8: 5873-5882. doi: http://dx.doi.org/10.5897/AJB09.1391.

Andres, J. and P. N. Bertin. 2016. The microbial genomics of arsenic. FEMS Microbiol. Rev. 40: 299-322. doi: https://doi. org/10.1093/femsre/fuv050.

Arnold, A. E. and F. Lutzoni. 2007. Diversity and host range of foliar fungal endophytes: Are tropical leaves biodiversity hotspots? Ecology 88: 541-549. doi: https://doi.org/10.1890/05-1459.

Castellanos-Mendoza, A., R. M. Castro-Acosta, A. Olvera, G. Zavala, M. Mendoza-Vera, E. García-Hernández, A. Alagón, M. A. Trujillo-Roldan, and N. A. Valdez-Cruz. 2014. Influence of $\mathrm{pH}$ control in the formation of inclusion bodies during production of recombinant sphingomyelinase-D in Escherichia coli. Microb. Cell Fact. 13: 137. doi: https://doi.org/10.1186/ s12934-014-0137-9.

Cheung, K. H. and J. D. Gu. 2007. Mechanism of hexavalent chromium detoxification by microorganisms and bioremediation application potential: A review. Int. Biodeteriorat. Biodegradat. 59: 8-15. doi: https://doi. org/10.1016/j.ibiod.2006.05.002.
Corrales-Pérez, D. y F. Martín-Romero. 2013. Evaluación de la peligrosidad de jales de zonas mineras de Nicaragua y México, y alternativas de solución. Bol. Soc. Geol Mex. 65: 427-446.

Costa, A. C.A. and F. P. Duta. 2001. Bioaccumulation of copper, zinc, cadmium and lead by Bacillus sp., Bacillus cereus, Bacillus sphaericus and Bacillus subtilis. Braz. J. Microbiol. 32: 1-5. doi: http://dx.doi.org/10.1590/S1517-83822001000100001.

Drewniak, L. and A. Sklodowska. 2013. Arsenic-transforming microbes and their role in biomining processes. Environ. Sci. Pollut. Res. 20: 7728-7739. doi: https://doi.org/10.1007/ s11356-012-1449-0.

El Baz, S., M. Baz, M. Barakate, L. Hassani, A. El Gharmali, and B. Imziln. 2015. Resistance to and accumulation of heavy metals by actinobacteria isolated from abandoned mining areas. Sci. World J. Article ID 761834. doi: https://doi. org/10.1155/2015/761834.

Gómez-Bernal, J. M., J. Santana-Carillo, F. Romero-Martin, M. A. Armienta-Hernández, O. Morton-Bermea y E. Aurora RuizHuerta. 2010. Plantas de sitios contaminados con desechos mineros en Taxco, Guerrero, México. Bol. Soc. Bot. Méx. 87: 131-133. doi: https://doi.org/10.17129/botsci.323.

Guerrero-Jiménez, G., G. Zavala-Padilla, M. Silva-Briano, and R. Rico-Martínez. 2013. Morphology and ultrastructure of the freshwater rotifer Brachionus bidentatus (Monogononta: Brachionidae) using scanning and transmission electron microscopy. Rev. Biol Trop. 61: 1737-1745. doi: https://doi. org/10.15517/rbt.v61i4.12818.

Hassan, S. H. A., R. N. N. Abskharon, S. M. F. Gad El-Rab, and A. A. M. Shoreit. 2008. Isolation, characterization of heavy metal resistant strain of Pseudomonas aeruginosa isolated from polluted sites in Assiut city, Egypt. J. Basic Microbiol. 48: 168-76. doi: https://doi.org/10.1002/jobm.200700338.

Heck, K., É. G. De Marco, M. W. Duarte, S. P. Salamoni, and S. Van Der Sand. 2015. Pattern of multiresistant to antimicrobials and heavy metal tolerance in bacteria isolated from sewage sludge samples from a composting process at a recycling plant in southern Brazil. Environ. Monit. Assess. 187: 328. doi: https://doi.org/10.1007/s10661-015-4575-6.

Hoffman, M. T. and A. E. Arnold. 2010. Diverse bacteria inhabit living hyphae of phylogenetically diverse fungal endophytes. Appl. Environ. Microbiol. 76: 4063-4075. doi: https://doi. org/10.1128/AEM.02928-09.

Ji, G. and S. Silver. 1992. Regulation and expression of the arsenic resistance operon from Staphylococcus aureus plasmid pI258. J. Bacteriol. 174: 3684-3694. doi: https://doi.org/10.1128/ jb.174.11.3684-3694.1992.

Maddison, D. R. and W. P. Maddison. 2005. ChromaSeq module. Mesquite: A modular system for evolutionary analysis. Version 1.06. http://mesquiteproject.org. (Consulta: abril 15, 2019).

Maddison, W. P. and D. R. Maddison. 2009. Mesquite: A modular system for evolutionary analysis. Version 2.6. http:// mesquiteproject.org/. (Consulta: abril 15, 2019).

Marrero-Coto, J., A. Díaz-Valdivia y O. Coto-Pérez. 2010. Mecanismos moleculares de resistencia a metales pesados en las bacterias y sus aplicaciones en la biorremediación. Rev. CENIC Cienc. Biol. 41: 67-78.

Martínez, A., M. Cruz, O. Veranes, M. E. Castro Carballo, I. Salgado, S. C. Olivares, L. Lima y D. K. Rodríguez. 2010. Resistencia a antibióticos y a metales pesados en bacterias aisladas del río Almendares. Rev. CENIC Cienc. Biol. 41: 1-10. 
Moraga, R., C. Merino y M. A. Mondaca. 2003. Resistencia a metales pesados en bacterias aisladas de la bahía de Iquique. Investig. Mar. 31: 91-95. doi: http://dx.doi.org/10.4067/S071771782003000100010 .

Mosa, K. A., I. Saadoun, K. Kumar, M. Helmy, and O. P. Dhankher. 2016. Potential biotechnological strategies for the cleanup of heavy metals and metalloids. Front. Plant Sci. 7: 303. doi: https://doi.org/10.3389/fpls.2016.00303.

Nunes, C. I., J. L. A. Brás, S. Najmudin, J. J. Moura, I. Moura, and M. S. Carepo. 2014. ArsC3 from Desulfovibrio alaskensis G20, a cation and sulfate-independent highly efficient arsenate reductase. J. Biol. Inorg. Chem. 19: 1277-1285. doi: https:// doi.org/10.1007/s00775-014-1184-8.

O'Brien, S. and A. Buckling. 2015. The sociality of bioremediation Hijacking the social lives of microbial populations to clean up heavy metal contamination. EMBO reports 16: 1241-1245. doi: https://doi.org/10.15252/embr.201541064.

Oves, M., M. S. Khan, and A. Zaidi. 2013. Chromium reducing and plant growth promoting novel strain Pseudomonas aeruginosa OSG41 enhance chickpea growth in chromium amended soils. Eur. J. Soil Biol. 56: 72-83. doi: https://doi.org/10.1016/j. ejsobi.2013.02.002.

Pandey, N. and R. Bhatt. 2015. Arsenic resistance and accumulation by two bacteria isolated from a natural arsenic contaminated site. J. Basic Microbiol. 55: 1275-1286. doi: https://doi. org/10.1002/jobm.201400723.

Poornima, K., L. Karthik, S. P. Swadhini, S. Mythili, and A. Sathiavelu. 2010. Degradation of chromium by using a novel strains of Pseudomonas species. J. Microbiol. Biochem. Technol. 2: 95-99. doi: https://doi.org/10.4172/19485948.1000031.

Román-Ponce, B., J. Ramos-Garza, M. S. Vásquez-Murrieta, F. N. Rivera-Orduña, W. F. Chen, J. Yan, P. Estrada-de los Santos, and E. T. Wang. 2016. Cultivable endophytic bacteria from heavy metal (loid)-tolerant plants. Arch. Microbiol. 198: 941956. doi: https://doi.org/10.1007/s00203-016-1252-2.

Saitou, N. and M. Nei. 1987. The neighbor-joining method: A new method for reconstructing phylogenetic trees. Mol. Biol. Evol. 4: 406-425. doi: https://doi.org/10.1093/oxfordjournals. molbev.a040454.

Saltikov, C. W. and B. H. Olson. 2002. Homology of Escherichia coli $\mathrm{R} 773$ ars A, arsB, and arsC genes in arsenic-resistant bacteria isolated from raw sewage and arsenic-enriched creek waters. Appl. Environ. Microbiol. 68: 280-288. doi: https:// doi.org/10.1128/AEM.68.1.280-288.2002.
Schlatter, D. C., M. G. Bakker, J. M. Bradeen, and L. L. Kinkel. 2015. Plant community richness and microbial interactions structure bacterial communities in soil. Ecology 96: 134-142. doi: https://doi.org/10.1890/13-1648.1.

Simeonova, D. D., D. Liévremont, F. Lagarde, D. A. E. Muller, V. I. Groudeva, and M. C. Lett. 2004. Microplate screening assay for the detection of arsenite-oxidizing and arsenate reducing bacteria. FEMS Microbiol. Lett. 237: 249-253. doi: https://doi. org/10.1111/j.1574-6968.2004.tb09703.x.

Talavera-Mendoza, O., M. A. Armienta-Hernández, J. GarcíaAbundis, and N. Flores-Mundo. 2006. Geochemistry of leachates from the El Fraile sulfide tailings piles in Taxco, Guerrero, southern Mexico. Environ. Geochem. Health 28: 243-255. doi: https://doi.org/10.1007/s10653-005-9037-6.

Talavera-Mendoza, O., M. Yta, R. Moreno-Tovar, A. DótorAlmazán, N. Flores-Mundo, and C. Duarte-Gutiérrez. 2005. Mineralogy and geochemistry of sulfide-bearing tailings from silver mines in the Taxco, Mexico area to evaluate their potential environmental impact. Geofís. Int. 44: 49-64.

Tchounwou, P. B., C. G. Yedjou, A. K. Patlolla, and D. J. Sutton. 2012. Heavy metals toxicity and the environment. Exp. Suppl. 101: 133-164. doi: https://doi.org/10.1007/978-3-7643-83404_6.

Toribio-Jiménez, J., M. A. Rodríguez-Barrera, A. M. Valdez-Lucena, A. Barrera-Flores, D. Segura, V. Wilson-Corral, E. FloresAlfaro, and Y. Romero. 2014. Production of biosurfactants by bacteria isolated from a mine tailing zone in Southern Mexico and their resistance to heavy metals. J. Bacteriol. Res. 6: 2331. doi: https://doi.org/10.5897/JBR2014.0128.

Verma, T., S. K. Garg, and P.W. Ramteke. 2009. Genetic correlation between chromium resistance and reduction in Bacillus brevis isolated from tannery effluent. J. Appl. Microbiol. 107: 14251432. doi: https://doi.org/10.1111/j.1365-2672.2009.04326.x.

Weisburg, W. G., S. M. Barns, D. A. Pelletier, and D. J. Lane. 1991. 16S Ribosomal DNA Amplification for phylogenetic study. J. Bacteriol. 173: 697-703. doi: https://doi.org/10.1128/ jb.173.2.697-703.1991.

Yang, Y., S. Wu, R. M. Lilley, and R. Zhang. 2015. The diversity of membrane transporters encoded in bacterial arsenic-resistance operons. PeerJ. 3: e943. doi: https://doi.org/10.7717/peerj.943.

Yu, X., Y. Li, C. Zhang, H. Liu, J. Liu., W. Zheng, X. Kang, X. Leng, K. Zhao, Y. Gu, X. Zhang, Q. Xiang, and Q. Chen. 2014. Culturable heavy metal-resistant and plant growth promoting bacteria in V-Ti magnetite mine tailing soil from Panzhihua, China. Plos One 9: e106618. doi: https://doi.org/10.1371/ journal.pone.0106618. 